\title{
Special Issue on the 1st China Fusion Energy Conference
}

\author{
Xuru Duan ${ }^{1} \cdot$ Guangnan $\mathrm{Luo}^{2} \cdot \mathrm{Min} \mathrm{Xu}^{1} \cdot \mathrm{Xiaogang} \mathrm{Wang}^{3} \cdot$ Yugang Wang $^{4}$ \\ Published online: 8 February 2021 \\ (C) The Author(s), under exclusive licence to Springer Science+Business Media, LLC part of Springer Nature 2021
}

The 1st China Fusion Energy Conference (CFEC), was held in Leshan, China on 25-27 November 2019. With around 1000 attendees from 130 institutions, the conference was a great success. The purpose of CFEC is to exchange ideas among researchers in Chinese fusion community, including research institutes, universities and industries. During the conference, participants presented the latest progress in fusion energy research, to promote Chinese participation in the construction, operation and experimental planning of ITER, as well as to support the design and key technology development of fusion reactors.

We are delighted to introduce this Special Issue which gathers revised and extended versions of some selected papers of the 1st CFEC. With ITER successfully entering the assembly phase, an abundance of engineering technologies and physics research are beginning to bear fruitful results. Nevertheless, to achieve the scientific goals of ITER, challenges remain in diagnostics, plasma control technology, theory and modelling and materials among other topics.

This special issue includes 20 articles, which present some latest results on experiment, engineering, materials, theory and modelling. The articles are placed in the following order: Experiments, Engineering and Materials, Theory and Modeling. We are grateful to the authors for their contributions.

Publisher's Note Springer Nature remains neutral with regard to jurisdictional claims in published maps and institutional affiliations.

Guest Editor: Xuru Duan

Associate Guest Editors: Guangnan Luo, Min Xu, Xiaogang

Wang, Yugang Wang

\author{
Xuru Duan \\ duanxr@swip.ac.cn \\ Southwestern Institute of Physics, Chengdu, China \\ 2 Institute of Plasma Physics, Chinese Academy of Sciences, \\ Hefei, China \\ 3 Harbin Institute of Technology, Harbin, China \\ 4 Peking University, Beijing, China
}

